\title{
Arbeitsmarkt für Mathematiker/innen
}

\author{
Günter Törner, Britta Berndtsen und Joyce Peters-Dasdemir
}

\begin{abstract}
Um die wesentlichen Aussagen dieser Arbeit vorwegzunehmen: Der Arbeitsmarkt für Absolventinnen und Absolventen ist auf den ersten Blick günstig - diese Rückmeldungen erhalten wir oft von unseren Ehemaligen. Dennoch entsprechen die Erwartungen unserer Studierenden nicht notwendigerweise passgenau den Anforderungen der Arbeitgeber, nicht zuletzt, weil das Berufsbild einer Mathematikerin bzw. eines Mathematikers eher unterentwickelt ist. Das erfordert von den Stellensuchenden Flexibilität und Offenheit, Interesse an interdisziplinären Anwendungen von Mathematik und auch die Bereitschaft, sich in Teams einzuarbeiten, auf jeden Fall auch der kompetente Umgang mit unbekannter Software. Wir müssen zur Kenntnis nehmen, dass sich unsere Absolventen im weiten Mathematik Arbeitsmarkt der 110 ooo Erwerbstätigen mit mathematischer Qualifikation letztlich ,verlieren' und sich zumeist Aufgaben zuwenden müssen, die während der Ausbildung kaum thematisiert wurden. Doch man hat solche Kompetenzen erworben.
\end{abstract}

Irritierende Informationen auf den ersten Blick

\subsection{Das grundsätzliche Interesse}

Es versteht sich von selbst, dass Wissenschaftler/innen kontinuierlich den Arbeitsmarkt ihrer Disziplin - über den Hochschulschulmarkt hinaus - im Blick haben. Zum einen, um schon im schulischen Bereich potentiellen Nachwuchs $\mathrm{zu}$ interessieren und zu werben, anderseits aber auch, um ihre Studierenden an den Hochschulen angemessen beraten und für den Arbeitsmarkt vorbereiten zu können.

Man muss allerdings seinen Kolleginnen und Kollegen zugestehen, dass es nicht einfach ist, darüber Zahlen und weitergehende Informationen zu erschließen. Natürlich kann man Google fragen und landet sehr schnell auf Seiten, die gesponsert werden und danach den aufrufenden Internetbesucher noch Wochen verfolgen. Bald wird offenbar, dass der Internetbetreiber für einen Finanzier neue Mitarbeiter/innen mit spezifischen Qualifikationen sucht. Der aktuelle Arbeitsmarkt im Ganzen - spezifiziert für mathematische Berufe - bleibt aber außerhalb des Blickwinkels.

\subsection{Wer beschäftigt sich mit Arbeitsmarktfragen?}

Die Bundesagentur für Arbeit ${ }^{1}$ (BA) (mit Hauptsitz in Nürnberg) mit mehreren Dependancen in Deutschland ist die erste Adresse, wenn es um Berufe und den Arbeitsmarkt geht. Gleichzeitig verweisen wir auf Daten des Statistischen Bundesamtes (Wiesbaden), deren wir uns bedient haben, um etwas über den mathematischen Arbeitsmarkt zu erfahren.

Die BA ist auch für die Herausgabe einer offiziellen Broschüre $^{2}$ über Akademiker-Berufe zuständig. Dem uns interessierenden Berufsfeld wird hier allerdings keine eigenständige Beschreibung gewidmet; Mathematiker werden hier zumeist im Zusammenhang mit Naturwissenschaftler/n/innen erwähnt. Unsere Nachfragen haben dazu geführt, dass sich dies mit der Neuauflage im Mai 2019 ändern wird. ${ }^{3}$

Früher - als HIS bekannt - beschäftigte sich auch das Deutsche Zentrum für Hochschul- und Wissenschaftsforschung $(\mathrm{DZHW})^{4}$, ein durch Bund und Länder gefördertes For- schungsinstitut mit Sitz in Hannover und Berlin, mit Arbeitsfragen. Wir zitieren von der Homepage der DZHW:

Als internationales Kompetenzzentrum der Hochschul- und Wissenschaftsforschung führt das DZHW Datenerhebungen und Analysen durch, erstellt forschungsbasierte Dienstleistungen für die Hochschul- und Wissenschaftspolitik und stellt der Scientific Community eine Forschungsinfrastruktur im Bereich der Hochschul- und Wissenschaftsforschung zur Verfügung. ${ }^{5}$

Auf den Seiten von DZHW findet man u. a. längerfristige Analysen über Arbeitsmarktkarrieren, zumeist aber unter Einbezug von Naturwissenschaftlerinnen und Naturwissenschaftlern.

Wir sollten auch das Institut für Arbeitsmarkt- und Berufsforschung ${ }^{6}$ (IAB) in Nürnberg erwähnen7 Das IAB sagt über sich:

Das Institut für Arbeitsmarkt- und Berufsforschung (IAB) erforscht den Arbeitsmarkt, um politische Akteure auf allen Ebenen kompetent zu beraten. Ökonomen, Soziologen, Wissenschaftlerinnen und Wissenschaftler weiterer sozialwissenschaftlich und methodisch ausgerichteter Disziplinen schaffen durch exzellente, national wie international vernetzte Forschung die Basis für eine empirisch informierte Arbeitsmarktpolitik.

$\mathrm{Zu}$ guter Letzt sollten wir darauf hinweisen, dass sich auch das Statistische Bundesamt mit Arbeitsmarktdaten beschäftigt. Jährlich veröffentlicht das Statistische Bundesamt den Bildungsstand der Bevölkerung ([6]). Wir beziehen uns auf den aktuellen Bericht ${ }^{8}$ aus dem Jahr 2017, der die Zahlen von 2016 auflistet. Der Bildungsstand stellt eines der Ergebnisse des Mikrozensus, eine Repräsentativstatistik über die Bevölkerung und den Arbeitsmarkt, dar, der jährlich mit einem Auswahlsatz von $1 \%$ der Bevölkerung über Befragungen durchgeführt wird. Folglich handelt es sich bei den im Mikrozensus ausgewiesenen Werten um hochgerechnete 
Zahlen, die daher lediglich als grobe Schätzwerte, z. B. für den gesamten Umfang der Erwerbstätigkeit, dienen. ${ }^{9}$

Erhobene Merkmale eines akademischen Abschlusses sind nunmehr Bachelor, Master, Diplom und Promotion; die Personengruppen werden nach ihrem Alter und Geschlecht differenziert, nämlich unter 30, 30-40, 40-50 und $50 \mathrm{u}$. m.

Die Bevölkerung wird in Erwerbs- und Nichterwerbspersonen (Personen, die am Erwerbsleben nicht teilnehmen können und/oder nicht teilnehmen wollen) unterteilt. Die Zahl der Erwerbspersonen ergibt sich als Summe aus den Erwerbstätigen (Personen, die mindestens einer Erwerbstätigkeit als beschäftigter Arbeitnehmer oder als Selbstständiger nachgehen) und den Erwerbslosen (Personen ohne Arbeit, die eine mindestens eine Stunde umfassende Tätigkeit suchen und für die Arbeitsaufnahme sofort zur Verfügung stehen). Erwerbslose können aufgrund der kleinen Zahlen nicht quantifiziert werden.

\subsection{Irritierende Zahlen: Nur 200 offene Stellen? 7oo Arbeitslose?}

Recherchiert man bei der Bundesagentur für Arbeit ${ }^{10}$ (BA), so berichtet die BA-Seite ${ }^{11}$ von lediglich knapp 200 Stellenangeboten im Jahr 2017, denen allein 700 Arbeitslose gegenüber stehen. Schließlich findet man auf der gleichen Seite, dass es anscheinend (nur) 9340 sozialversicherungspflichtig Beschäftigte in der Berufeauswahl $411 \mathrm{Ma}$ thematik und Statistik gibt, m.a.W. scheint es um einen sehr kleinen Arbeitsmarkt zu gehen. Alles auf den ersten Blick - wie im Titel ausgeführt - irritierend, drängen doch mehr als 2000 Qualifizierte jährlich in diesen Arbeitsmarkt.

In den vergangenen Jahren hatte der erste Autor in den Mitteilungen der Deutschen Mathematiker-Vereinigung drei Publikationen $([1-3])$ vorgelegt, die sich mit dem Arbeitsmarkt für Mathematiker/innen beschäftigten. Die letzte Veröffentlichung liegt allerdings schon vier Jahre zurück und nicht weniges hat sich in den letzten vier Jahren verändert, nicht zuletzt die Bachelor-Master-Reform des Studiums, die generellen Absolventenzahlen usw. Die Studien lassen nun zwei Zeitpunkte zu, an denen man (theoretisch) in den Arbeitsmarkt eintreten kann.
Zuversichtlichere Daten - oder: Was man wissen sollte

2.1 Terminologie

Im Jahr 2010 wurde von der Bundesagentur (BA) eine neue Klassifikation ${ }^{12}$ der Berufe, kurz KldB 2010, eingeführt, die das Fach Mathematik zusammen mit der Nachbardisziplin Statistik in der eigenständigen Berufsgruppe 411 führt und wie folgt in weitere vierstellige Berufsuntergruppen differenziert:

- 4110 Berufe in der Mathematik (ohne Spezialisierung),

- 4111 Berufe in der Statistik,

- 4118 Berufe in der Mathematik (sonstige spezifische Tätigkeitsangabe) und

- 4119 Führungskräfte Mathematik und Statistik.

Schulische Lehrkräfte werden hier nicht erfasst. Durch dieses neue, wesentlich überschaubarere Klassifikationsschema sind wir eigentlich in der guten Ausgangslage, explizit mathematische Berufe und zugehörige Parameter zu identifizieren. Andererseits sind die Zahlen in dieser Berufsgruppe klein, so dass sehr oft in den öffentlichen Statistiken weiterhin die Mathematiker/innen mit Physiker/n/innen resp. Naturwissenschaftler/innen gemeinsam geführt werden, was nicht sehr hilfreich ist.

\subsection{Mathematische Qualifikationen von Erwachsenen in Deutschland}

Grundsätzlich ist es interessant zu wissen, wie viele Mathematiker/innen in Deutschland, unterwegs' sind. Anders formuliert: Wie viele Personen besitzen einen akademischen Bildungsabschluss nach einem erfolgreichen Mathematikstudium? Das ist eine Frage nach einem spezifischen Bildungsstand der Bevölkerung, die uns das Statistische Bundesamt ([6, S. $139 \mathrm{ff}]$.$) aktuell beantworten kann.$

In Tabelle 1 geben wir die Ergebnisse des Mikrozensus wieder. Ein ,/' bedeutet, dass das hochgerechnete Jahresergebnis im Mikrozensus kleiner als 5000 ist und damit nicht ausgewiesen wird. Leere Tabellenfelder entstehen dadurch, dass die Zahlen der Frauen nicht bekannt sind und somit auch keine Rückschlüsse auf die Zahlen der Männer gezogen werden können. Da außerdem die Zahl der erwerbslo-

Tabelle 1. Bevölkerung 2016 mit einem akademischen Abschluss in Mathematik in 1000

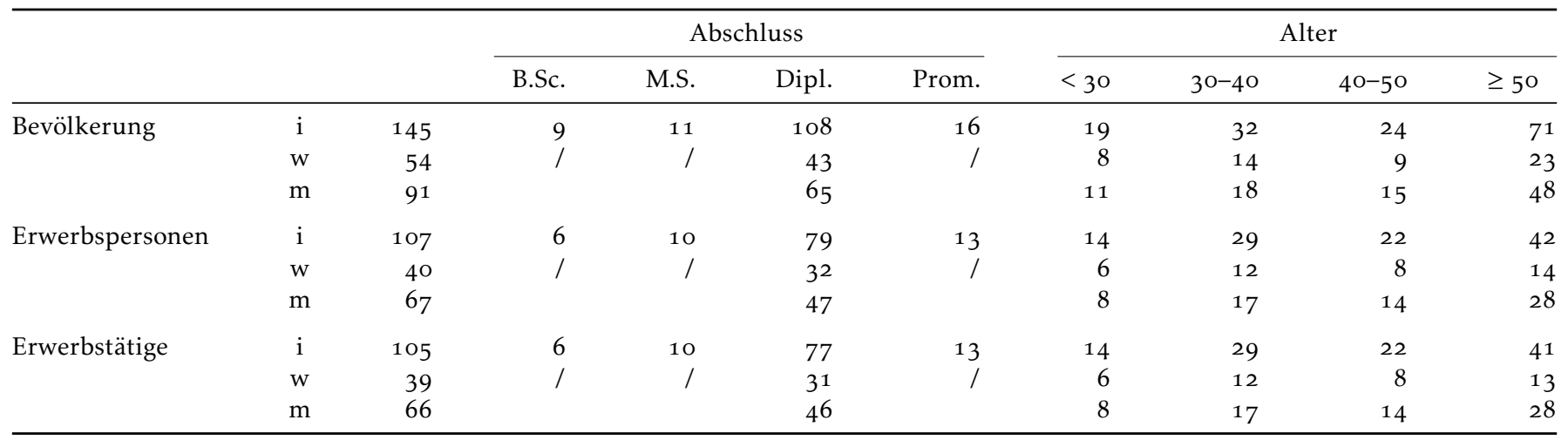


sen Mathematiker/innen zu gering $(<5000)$ ist, kann dieser Wert über den Mikrozensus leider nicht ermittelt werden. Die Werte in der Tabelle 1 bieten aber ein Indiz dafür, dass die Zahl der Arbeitslosen mit einer mathematischen Qualifikation im Jahr 2016 bei ca. 2000 lag.

Wir wollen die einzelnen Werte in der Tabelle nicht kommentieren, vielleicht merke man sich die Zahlen:

- Es befinden sich rund 107.00o akademisch mathematisch qualifizierte Personen im bundesdeutschen Arbeitsmarkt.

- Die Frauenquote für berufstätige Mathematikerinnen beträgt rund $37 \%$.

\subsection{Sozialversicherungspflichtige Mathematiker/innen}

Die Werte im Mikrozensus (vgl. Tabelle 1) beruhen auf den expliziten Angaben der Befragten; sie zählen also jene Personen, die sich als Mathematiker/innen betrachten. Anders sieht es bei den sozialversicherungspflichtig Beschäftigten aus der Berufsgruppe 411 aus; die Informationen stammen aus den Meldungen der Arbeitgeber. Es ist schnell gesagt, wer nicht sozialversicherungspflichtig ist: Beamte, Richter, Soldaten; Selbständige und mithelfende Familienangehörige; Freiberufler. Wenn diese einen mathematischen Beruf ausüben, sollte diese Zahl wohl nicht fünfstellig sein, meldet doch ([7]) im Jahr 2017 rund 6ooo hauptamtliche beamtete Mathematiker/innen.

Wenn nun die BA die sozialversicherungspflichtigen erwerbstätigen Mathematiker/innen erhebt, so beruft sich die Behörde auf die Meldungen ihrer Arbeitgeber gemäß den Charakteristiken der Berufsgruppe 411. Einer Zusammenstellung ${ }^{13}$ des IAB können wir die Zahl der Sozialversicherungspflichtigen in der Berufsgruppe Mathematik und Statistik entnehmen:

Tabelle 2. Sozialversicherungspflichtigen in der Berufsgruppe Mathematik und Statistik

\begin{tabular}{llllll}
\hline Jahr & 2013 & 2014 & 2015 & 2016 & 2017 \\
Anzahl & 9.654 & 9.477 & 9.299 & 9.226 & 9.322 \\
\hline
\end{tabular}

Auch gibt uns die Statistik Informationen, was die Verteilung auf die Branchen anbetrifft. Wir beschränken uns auf das Jahr 2017, müssen uns aber eingestehen, dass die Quoten wenig aussagefähig sind.

- Produzierendes Gewerbe 8,6\%

- Dienstleistungssektor 91,3\% mit den Unterkategorien

- Kredit- und Versicherungsgewerbe 30,3\%

- Ingenieurbüros, Rechtsberatung, Werbung 14,6\%

- Öffentliche Verwaltung, Sozialversicherung 9,9\%

- Übrige Dienstleistung 23,8\%

Betrachtet man die Tendenz der sozialversicherungspflichten Beschäftigungen im Berufsfeld Mathematik, so deuten die vorgelegten Zahlen eher eine Stagnation an. Dies gilt allerdings nicht für den IT-Arbeitsmarkt, wie man ([8], S. 15) entnehmen kann:

Am deutlichsten zeigte sich 2017 das Beschäftigungswachstum sowohl relativ als auch absolut in den ITBerufen. Hier hat die Zahl der sozialversicherungs- pflichtigen Arbeitsplätze gegenüber dem Vorjahr um 16 ooo oder sieben Prozent zugelegt.

\subsection{Mathematikabsolventen pro Jahr}

Der Mikrozensus vermittelt uns die Größenordnung der im Erwerbsleben stehenden mathematisch-qualifizierten Personen; über die Zahlen von Destatis können wir nun den jährlichen Zuwachs abschätzen, in dem (vereinfachend) die Zahl der erfolgreichen Master/Diplom-Prüfungen zählen (vgl. Tabelle 3). Dass sicherlich eine mittlere dreistellige Zahl von Bachelor-Absolventen in den Arbeitsmarkt wechselt, stärker von Fachhochschulen als den Universitäten kommend, wollen wir hier ignorieren.

Knapp 2200 akademisch-qualifizierte Mathematiker und Mathematikerinnen diffundieren in den Arbeitsmarkt, zum geringen Teil auch in den hochschulinternen. Diese Zahl steht erneut im starken Kontrast zu den durch die BA rund 200 gemeldeten Stellen.

\subsection{Erkärungsversuche für die enormen Unterschiede}

Die Zahlen der mathematisch-qualifizierten Erwerbspersonen (laut Mikrozensus und laut Bundesagentur) weichen erheblich voneinander ab; insofern können wir wohl unterstellen, dass diese Personen zwar zum großen Teil ebenfalls sozialversicherungspflichtig beschäftigt, nicht aber in die Berufsgruppe 411 der Mathematiker/innen eingeordnet sind. Kollege Günter M. Ziegler sprach einmal von einem ,Bermuda-Dreieck', in dem mehr als 90 \% der Mathematiker/innen zunächst statistisch verschwinden, aber sofort in einer anderen Berufsgruppe wieder auftauchen. Das belegen viele subjektive Beobachtungen und schließlich auch die Interviewserie „Mathematik studiert - und dann?" in den Mitteilungen (vgl. Abschnitt 3).

Die Bundesagentur meldet zwar 8oo Arbeitslose; wie man uns sagte, scheinen nicht wenige individuelle Problemfälle darunter zu sein, die nicht primär in der mathematischen Qualifikation des Arbeitssuchenden begründet sind. Vermutlich lebt statistisch bei diesen Personen die ursprüngliche Qualifikation - Mathematikstudium - auf, wenn sie erfasst werden und so werden sie der Berufsgruppe 411 (wieder) zugeschlagen.

Im Mikrozensus sind die Arbeitslosenzahlen so klein, dass sie nicht erfasst werden können.

\section{Empfehlungen von im Berufsleben stehenden Mathematiker/innen}

Unser Text sollte wohl deutlich gemacht haben, dass das nirgendwo befriedigend erklärte Berufsbild eines Mathematikers ein Manko darstellt. Zugegeben: Es ist nicht einfach, dieses Berufsbild zu beschreiben, weil die Mathematikausbildung eine sehr breite Qualifizierung anstrebt und zugleich Mathematiker/innen zunehmend in bisher unbekannten Anwendungsfeldern eingesetzt werden. Auf jeden Fall sollte er herausstellen, dass die Berufsanfänger schon lange nicht mehr fast ausschließlich in der Versicherungsmathematik eingesetzt werden. 


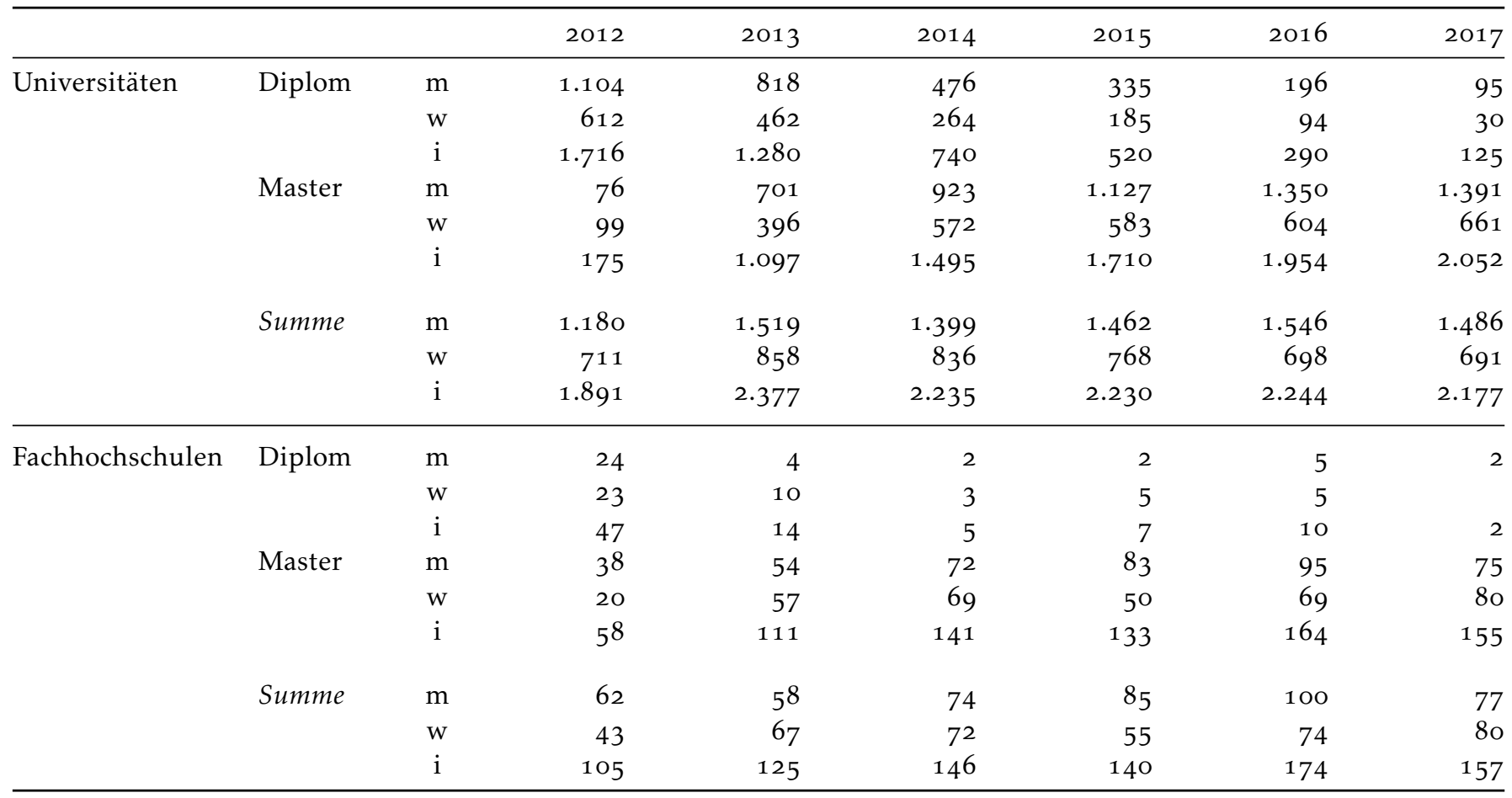

Einen wertvollen Beitrag leistet die Artikelserie „Mathe studiert - und dann?" in den Mitteilungen der Deutschen Mathematiker-Vereinigung seit dem Jahr 2010. Wir haben diese interessanten Artikel quer gelesen und bündeln die dort erwähnten Argumente; wenn wir zitieren, bedienen wir uns Anführungszeichen und geben die Quelle explizit an. Ansonsten haben wir sinngemäß umformuliert. Man wünschte sich, dass einige dieser Aussagen im Studium vermittelt werden können.

Listet man die Tätigkeitsfelder von Mathematiker/innen auf, so stammen die interviewten Personen aus Wissenschaften und der Schule, Finanz- und Versicherungswirtschaft, der Fahrzeug- und Flugzeugbranche, Unternehmensberatungen, Schulbuchverlagen, Programmierung und Softwareentwicklung (z. B. auch Computerspiele, Filmanimationen), Ingenieurunternehmen, Aktuar, Projektmanagement, Spiel- und Leistungsanalyse, Informationstechnik, Gesundheitssektor, Dokumentenanalyse (OCR, Ahnenforschung), Landschaftsökologie, der Start-Up-Branche (Buchverlag, Essensbranche, ...) und aus der Öffentlichkeitsarbeit.

Was die nie vollständig auflistbaren Tätigkeitsfelder anbetrifft, artikulieren einzelne folgende Texte:

- „Man kann Mathematik in so unglaublich vielen Bereichen anwenden, nicht nur im Banken- und Versicherungssektor. Aber man braucht eine gewisse Offenheit, um dorthin zu gelangen." (Mitteilungen 24-2 (2016))

- In der Versicherungsmathematik ist eine ergänzende Aktuar-Ausbildung fast unerlässlich.

- „Als Mathematiker bin ich (oft) ein Exot. Das merken Kollegen an meiner Arbeitsweise: Ich strukturiere meine
Aufgaben, Haushaltspläne und Analysen etwa, ganz anders als jemand mit einer rein geisteswissenschaftlichen Ausbildung. Ein Mathematiker ist in der Berufswelt nicht selten auf sich alleine gestellt." (Mitteilungen 24-4 (2016))

Vor allem werden Fähigkeiten zur „Kommunikation“ und „Kooperation “ herausgestellt:

- „Kommunikation ist sehr wichtig. In der Mathematik werden oft die Ergebnisse sehr hoch gehängt, aber was auch wichtig ist und oft vergessen wird, ist, dass diese Ergebnisse auch schlüssig und allgemein verständlich präsentiert werden müssen." (Mitteilungen 23-2 (2015))

- Ein Mathematiker muss mit anderen Naturwissenschaftlern und Ingenieuren kommunizieren können.

- „Was aber ganz wichtig ist: Kommunikationsfähigkeit. Die muss man in einem Unternehmen erst mal unter Beweis stellen, wenn man direkt von der Universität kommt. Gut kommunizieren zu können, das traut man promovierten Mathematikern nicht automatisch zu." (Mitteilungen 21-4 (2013))

- Dazu ist die Bereitschaft und Fähigkeit zur Teamarbeit unabdinglich.

- „Was die Mathematik angeht schon, aber auf das Arbeitsleben war ich überhaupt nicht vorbereitet. Ich hatte kaum Erfahrung mit Teamarbeit. Das habe ich erst hier gelernt." (Mitteilungen 24-3 (2016))

Explizit werden in den Interviews Kontakte zu Ingenieuren, Chemikern, der Pharmaindustrie, verschiedene Auslandskontakte, zu Regierungsorganisationen, IT-Experten, Lehrpersonen, Hochschulen, neben Informatikern auch häufig Physiker, Grafiker, Sportler (Vereinen), Techniker, Medizi- 
ner, Biologen, Umweltwissenschaftler, Geisteswissenschaftler, Elektrotechniker erwähnt.

Hinsichtlich der subjektiven Einstellungen betonen die Interviewten:

- Passion für Mathematik ist nicht hinderlich. Passion, sich Problemen auszusetzen.

- Man sollte offen für Anwendungsbezüge sein.

- „Im Prinzip reicht ein Mathematikstudium. Aber man sollte während des Studiums über den Tellerrand hinausschauen." (Mitteilungen 24-2 (2016))

- Nebenfachaffinität sollte nicht abschrecken.

- „Das können unmittelbar mathematische Fragestellungen sein, aber Bewerber sollten auch Interesse an gesellschaftlichen Fragestellungen haben." (Mitteilungen 23-1 (2015))

- „Ich würde sogar sagen, dass Mathematik ein Studium fürs Leben ist - selbst im Nebenfach." (Mitteilungen 24-4 (2016))

Zum Lernen im Mathematikstudium führen einzelne aus:

- Im Mathematikstudium sollte man lernen: Struktur im Denken, Abstraktionsvermögen und Präzision im Detail.

- „Auch in technologischen Zusammenhängen ist Beweisen angesagt: Wenn man nicht nachweisen kann, dass die Ansteuerung der Bremse durch das Radar wirklich immer funktioniert und dies auch schnell genug passiert, dann wird sich eine Versicherung möglicherweise fragen, ob sie die Haftung übernehmen kann." (Mitteilungen 25-4 (2017))

- Die Lösbarkeit eines Problems unter gewissen Prämissen zu beurteilen ist ein Thema, bei dem die Mathematik ihre Stärken ausspielen kann.

- „Ja, man lernt, Probleme einzuschätzen und sie zu visualisieren, um sie zu verstehen. Diese Schulung des Vorstellungsvermögens und das Wissen, welche Anschauungen angewendet werden können, um Probleme zu minimieren oder zu optimieren, das ist großartig." (Mitteilungen 24-4 (2016))

- „Vom Mathematik-Studium ist mir eine Art Gewissheit geblieben, dass vieles möglich ist, auch wenn ich die Lösung noch nicht kenne." (Mitteilungen 24-4 (2016))

- „Ich gehe ähnlich kritisch und strukturiert an ein Problem heran wie bei einem mathematischen Beweis, etwa indem ich alle Sonderfälle, alle Eventualitäten berücksichtige. Das sind mathematische Kompetenzen, die ich einbringe genauso wie das analytische Denken oder die Präzision im Denken und in der Ausführung." (Mitteilungen 23-4 (2015))

- „Ganz wichtig sind Programmierkenntnisse. Und damit meine ich nicht nur eine bestimmte Programmiersprache, sondern die Softwareentwicklung als Konzept. ... Als Algorithm-Engineer sollte man auf jeden Fall das Programmieren mögen, und ich profitiere heute davon, dass ich mich als Student an Projekten mit Industriepartnern beteiligt habe." (Mitteilungen 24-1 (2016))

- „Mit Stochastik sollten sie vertraut und in Informatik fit sein. Ganz allgemein ist algorithmisches Denken hilf- reich, weil man ein System von Regeln und Abläufen hat, das man gut verstehen muss." (Mitteilungen 23-3 (2015))

Sonstige berufsrelevante Erfahrungen:

- „Sich nicht durch Stellenausschreibungen entmutigen lassen: [...] man sieht nicht genau, welche Tätigkeit sich hinter einer Job-Beschreibung verbirgt. [...] Ich glaube Mathematiker lassen sich von den Job-Beschreibungen in den Stellenangeboten abschrecken. Die erscheinen ihnen $\mathrm{zu}$ weit weg von den Inhalten des Studiums." (Mitteilungen 22-2 (2014))

- Vorab-Kommunikation mit Firmen, die Mathematiker brauchen.

- „Ich kann nur dazu raten, schon während des Studiums Praktika zu absolvieren oder aber als Werkstudent zu arbeiten. [...] Auch direkte Kontakte zu Mitarbeitern des Unternehmens können bei der Suche nach einem Praktikumsplatz oder einer Stelle helfen. Solche Kontakte bekommt man zum Beispiel über Mentoring-Programme oder auch bei Firmenkontaktmessen." (Mitteilungen 20-4 (2012))

- „Ich kann jedem nur raten, schon als Werkstudent aktiv $\mathrm{zu}$ werden. Das war jedenfalls für mich der Schlüssel. Man hat die Chance, etwas auszuprobieren, kann erst mal irgendwo anfangen, und selbst wenn es einem nach einem halben Jahr nicht mehr gefällt, nimmt einem das keiner übel. Danach ist man auf jeden Fall schlauer." (Mitteilungen 24-3 (2016))

- Besuch von Jobmessen.

- „Schon während der Promotion war mir klar, dass ich die akademische Karriere nicht weiterverfolgen möchte." (Mitteilungen 23-4 (2015))

- Stellenanzeigen spielen auf dem Job-Markt eine untergeordnete Rolle, Interessenten sollten sich einfach bewerben.

Bleibt noch etwas zu tun?

Eigentlich könnte man den Text zufrieden zur Seite legen: Mathematiker/innen haben auf dem Arbeitsmarkt keine Schwierigkeiten unterzukommen, die Erwerbslosigkeit liegt bei 2,5\% auf einem Stand, der vernachlässigbar ist. Und dennoch stimmt es möglicherweise aufmerksame Leser nachdenklich.

Das Berufsbild eines/r Mathematiker/in ist unterentwickelt. Die Fachgesellschaften der Mathematik haben - nach Wissen der Autoren - nirgendwo eine umfassende Darstellung veröffentlicht, sieht man von dem verdienstvollen Büchlein [5] im Mathematikjahr 2008 einmal ab. Vermutlich wird den Anfängerstudierenden selten die enorme Einsatzbreite einer mathematischen Qualifikation aufgezeigt. Wenn man von der Bedeutung der Mathematik in der Versicherungsmathematik berichtet, sollte man insbesondere die zentrale Rolle einer (ergänzenden) Aktuarausbildung erwähnen.

Gleichwohl haben die neueren Herausgeber der Mitteilungen der Deutschen Mathematiker-Vereinigung dieses Defizit erkannt und die schon oben erwähnte, lesenswerte Serie un- 
ter der Überschrift „Mathe studiert - und dann?“ gestartet. Mitgliedern ist diese Serie über den Bezug der Mitteilungshefte zugänglich.

Ob man nicht auf den Homepages der DMV ${ }^{14}$, der Gesellschaft für Angewandte Mathematik und Mechanik ${ }^{15}$ (GAMM) und selbst jene der Deutschen Aktuarvereinigung ${ }^{16}$ (DAV) Studierende darüber informieren könnte? Es wäre ein wichtiger Beitrag zu einer Herausbildung eines Berufsbildes.

Die enorme Breite der Einsatzgebiete für mathematisch Qualifizierte erklärt möglicherweise, warum sich von den laut Mikrozensus 107 ooo Personen - eine stolze Zahl - in den Fachgesellschaften noch nicht einmal eine fünfstellige Zahl wiederfindet. Für Werbeaktionen wäre hier noch Luft nach oben ...

Ja, die Community der Mathematiker/innen sollte noch mehr über ihre Berufsfelder informieren.

\section{Anmerkungen}

1. www.arbeitsagentur.de/privatpersonen

2. tinyurl.com/ybexahgq

3. tinyurl.com/ybexah9q

4. www.dzhw.eu

5. Gregor Fabian beim DZHW war unseren Fragen gegenüber aufge-

schlossen.

6. bisds.iab.de

7. Eine hilfreiche Ansprechpartnerin war uns beim IAB Dagmar Heimbach.

8. tinyurl.com/yaw6zx4l

9. Für detailliertere Informationen über den Mikrozensus, siehe u. a. [1].

10. Wir danken Frau Kirsten Singer (BA Düsseldorf), die uns hilfreiche Hinweise gegeben und mehrfach unsere Fragen geduldig beantwortet hat.

11. tinyurl.com/yaxsryyq

12. tinyurl.com/y7 fkjvhe

13. tinyurl.com/y8e59lv3

14. www.mathematik.de

15.www.gamm-ev.de/index.php/de/

16. aktuar.de
Literatur

[1] Dieter, M. \& Törner, G. (2009): Zahlen rund um das Mathematikstudium - Teil 5: Zahlen zum Bildungsstand und zum Arbeitsmarkt. Mitteilungen der Deutschen Mathematiker-Vereinigung 17-2, S. 111-116.

[2] Dieter, M. \& Törner, G. (2009): Zahlen rund um das Mathematikstudium - Teil 6: Der Arbeitsmarkt für Mathematiker. Mitteilungen der Deutschen Mathematiker-Vereinigung 17-4, S. 247-252.

[3] Dieter, M. \& Törner, G. (2014): Der Arbeitsmarkt für Mathematiker - Teil 1: Mitteilungen der Deutschen Mathematiker-Vereinigung 22, S. $154-157$.

[4] Fabian, G.; Hillmann, J.; Trennt, F.; Briedis, K. 2016. Hochschulabschlüsse nach Bologna. Werdegänge der Bachelor- und Masterabsolvent(inn)en des Prüfungsjahrgangs 2013. Hannover: DZHW.

[5] Greuel, G.-M.; Remmert, R.; Rupprecht, G. (2008). MathematikMotor der Wirtschaft. Berlin: Springer.

[6] Statistisches Bundesamt (2017): Bildungsstand der Bevölkerung 2016. Wiesbaden: Destatis.

[7] Statistisches Bundesamt (2018): Bildung und Kultur - Personal an Hochschulen 2017. Fachserie 11 Reihe 4.4.

[8] Statistik der Bundesagentur für Arbeit Berichte: Blickpunkt Arbeitsmarkt-Akademikerinnen und Akademiker. Nürnberg, Mai 2018.

Prof. Dr. Günter Törner, Britta Berndtsen, Joyce Peters-Dasdemir

Universität Duisburg-Essen, Fakultät für Mathematik,

Thea-Leymann-Straße 9, 45117 Essen

guenter.toerner@uni-due.de

britta.berndtsen@uni-due.de

joyce.peters-dasdemir@uni-due.de

\footnotetext{
Günter Törner, ursprünglich in der Mathematik (Algebra und Diskrete Mathematik) und der Didaktik der Mathematik mit zahlreichen Forschungsbeiträgen zu Hause, genießt das Emeritus-Leben, allerdings weiterhin mit vollem Tagesplan. Seine fast sechsjährige Tätigkeit als Ombudsman der Universität Duisburg-Essen für, Gute wissenschaftliche Praxis' hat seinen Blick auf die Forschungsrealitäten verändert. Weiterhin finden europäische Lehrer-Weiterbildungszentren und auch die Förderung der MINT-Ausbildung in einem ERASMUS+ Projekt seine Aufmerksamkeit.

Britta Berndtsen ist nichtwissenschaftliche Mitarbeiterin an der Fakultät für Mathematik der Universität Duisburg-Essen und insbesondere für den Bereich ,Gute wissenschaftliche Praxis' (GwP) zuständig.

Joyce Peters-Dasdemir ist wissenschaftliche Mitarbeiterin der Abteilung A des Deutschen Zentrums für Lehrerbildung Mathematik (DZLM) an der Universität Duisburg-Essen. Sie ist an Multiplikator- und Weiterbildungsprogrammen für Lehrkräfte beteiligt und auf dem Gebiet der Entwicklungskoordination tätig. Ihre Forschungsinteressen sind die Entwicklung und Implementierung von PD-Zentren, konzeptionelles Wissen und Überzeugungen von Lehrkräften zu digitalen Medien und die Beschreibung von Professionalisierungsprozessen von Multiplikatorinnen und Multiplikatoren.
} 